Article

\title{
Rheology of Un-Sieved Concentrated Domestic Slurry: A Wide Gap Approach
}

\author{
Adithya Krishnan Thota Radhakrishnan ${ }^{1, *}$, Jules van Lier $^{1}$ and Francois Clemens ${ }^{1,2}$ (]) \\ 1 Department of Water Management, Delft University of Technology, Civil Engineering and Geosciences, \\ P.O. Box 5, 2600 AA Delft, The Netherlands; J.B.vanLier@tudelft.nl (J.L.); F.H.L.R.Clemens@tudelft.nl (F.C.) \\ 2 Department of Industrial Hydrodynamics, Deltares, $2600 \mathrm{MH}$ Delft, The Netherlands \\ * Correspondence: a.k.thotaradhakrishnan@tudelft.nl; Tel.: +31-634-336-009
}

Received: 30 July 2018; Accepted: 14 September 2018; Published: 20 September 2018

check for updates

\begin{abstract}
Information on the rheology of domestic slurries is essential in designing pipeline transportation in novel sanitation systems. As concentrated slurries in their original collected state have wide particle size distribution, with particles up to $2 \mathrm{~mm}$, a wide gap rheometer is used to acquire the rheograms. Rheograms obtained from a wide gap rheometer require a method to convert the rotational velocity to the shear rate, and this method must be robust to noisy data and yield stress in the slurry. For this purpose, a Tikhonov regularisation method is chosen as it suits the criteria the best. Using this, the rheograms are obtained for various total suspended solids (TSS) concentrations of slurries. A Herschel-Bulkley rheological model is used to represent the rheology of the slurries. The influence of the change in concentration of the slurries is represented through its influence on the Herschel-Bulkley parameters. The consistency index $K$ exponentially increases with the concentration. The yield stress $\tau_{y}$, is 0 at low concentrations, and above $2.0 \%$ TSS (wt./wt.) exponentially increases with the concentration. The behaviour index $n$, is 1 at low concentrations, and above $2.6 \%$ TSS (wt./wt.) it decreases in an inverse power law with the concentration to reach a sort of plateau.
\end{abstract}

Keywords: concentrated domestic slurry; un-sieved slurry; rheology; Couette inverse problem; concentration influence

\section{Introduction}

Novel sanitation systems that incorporate source separation, vacuum toilets, and food waste disposers have shown promising results regarding nutrient and resource recovery. Although these claims are made on the prospect that low dilution is beneficial to the treatment process, it ignores the hydrodynamic aspects that are incurred in transporting the waste from the collection point to the treatment plant. This is to say that the minimum required dilution (i.e., minimum flush water quantity) for the transport of these slurries is still not known. Therefore, to realise these concepts in scale, a holistic assessment is required which includes collection, transportation, and treatment. Although collection and treatment options have been largely studied, transport of these slurries have been largely overlooked and will be the focus of this study.

Concentrated domestic slurries (CDS) arising from the novel sanitation systems are likely to contain solid kitchen waste along with cohesive particles like faeces, fibres such as toilet paper, and urine as liquids. The use of vacuum toilets and source separation will make them less dilute. To evaluate the transport concepts of these slurries their rheological properties are required [1-3]. The rheological properties are measured using rheometers.

Rheometers are commonly used in measuring the rheological properties of sludge and, are placed into two general categories: rotational rheometer and capillary rheometers. Rheology studies are commonly 
limited by the measuring instrument and its advantages and disadvantages determine the choice of rheometer to be considered. Capillary rheometers measure the rheology of a fluid through flow of the fluid in a pipe. Since the pressure drives the fluid through a pipe, the shear-rate varies along the radial direction and the flow is non-homogenous, therefore limiting the rheometer to study only steady stress-shear rate behaviour for time independent fluids [4]. Rotational rheometers on the other hand are able to provide a constant (or nearly so) shear rate throughout the system employed. Very narrow geometries of a narrow gap coaxial cylinder or a cone-plate system provide this; they can be limiting to multiphase fluids with particulates (discussed later) which preclude the use of narrow systems [4]. Although using rheometers are a conventional means to measure the rheology of a fluid, other non-conventional methods have been applied as well. These non-conventional methods usually stem from the limitations of rheometric techniques, rheological measurements from the engineering application itself, and the need for inline measurements. Some of the non-conventional techniques to estimate the rheology are through free surface velocity of the fluid flow [5], flow from spreading of a fluid from gravity currents [6], flow in a narrow channel [7], and ultrasound image velocimetry of flow in pipes [8].

Rotational rheometers, particularly the Couette-flow type, have traditionally been used to study complex fluids and have become widely accepted in recent years as the most common class of rheometer utilised in sludge rheology [9]. Studying rheology using a Couette-flow rheometer is limited by the maximum particle size of the constituents in the slurry. Wazer [10] recommends the minimum gap between the cylinders to be 10 times that of the maximum particle size in the slurry. This is to guarantee a shear flow of at least ten layers which ensures flow continuum. Despite the acknowledgment of the necessity of such a gap [4,11,12], only a few studies (rheology of similar slurries) have measured the slurry particle size for the choice of rheometer geometry $[13,14]$. This lack of attention to the particle size and distribution was also reported in the review work of Ratkovich [15]. For digested and waste activated sludge, the gap size constraint is less critical, because the range of the particle size is in order of a few hundred micrometres; therefore, a common narrow gap configuration is acceptable. However, this is not the case for raw sewage sludge where particles as large as a few millimetres have been reported $[16,17]$. To avoid the problem due to the size, it was suggested to work with sieved sludge where particles exceeding a certain diameter were removed $[16,17]$. However, it was acknowledged that for some samples, i.e., raw sewage sludge and the organic fraction of municipal solid waste, the sieved sludge did not represent the rheological behaviour of the whole sample, since more than $30 \%$ of the total solids were removed after sieving. Of the many articles published to characterise the rheology of wastewater slurries, to the author's knowledge, none characterise concentrated domestic slurry. For faecal sludge, the only article addressing its rheology by Woolley [18] is not comprehensive enough and is regarded to be at most a preliminary analysis. Therefore, this paper presents a detailed rheological study of un-sieved concentrated domestic slurry in a wide gap Couette-flow Searle type rheometer (MCR 302 Anton Paar, Graz, Austria).

There are several problems that need to be overcome in studying the rheology of un-sieved slurry. Firstly, the gap-size constraint due to the large particles requires a wide gap, where the general assumptions to obtain the shear-rate from the rotation speed are not valid as they are for a conventional narrow gap. And secondly, the noise in the data requires the use of particular procedures (detailed in later sections) in obtaining the underlying rheogram. This paper details a methodology to overcome these difficulties and to measure the rheology of CDS.

\section{Rheometry}

Couette-flow rheometers consist of two coaxial cylinders with the annulus between them filled with the fluid under study. In particular, a Searle-type system has the inner cylinder rotating and the outer one stationary. To obtain the rheogram (shear-stress vs. shear-rate curve) in a Searle system, the torque and rotational velocity of the inner cylinder is measured. The shear-stress can be calculated directly from the torque. But converting the rotational velocity to the shear-rate is not straightforward since it is an ill-posed inverse problem commonly referred to as the Couette inverse problem [19-22], 
and has been investigated by many researchers in the past decades. This conversion will be the focus of this study applying it to CDS.

\subsection{Couette Inverse Problem}

A Couette-flow Searle type rheometer consists of two coaxial cylinders where the inner cylinder (or bob) of radius $R_{i}$ and length $h$ rotating at a constant rotational speed of $\Omega$ and a stationary outer cylinder (or cup) of radius $R_{o}$, shown in Figure 1a. Balancing the forces yields the opposing torque (M) exerted on the bob by the fluid:

$$
M=2 \pi h r^{2} \tau
$$

$r$ being any location in the annulus, $R_{i} \leq r \leq R_{o}$. The shear-stress applied on the fluid at the bob is:

$$
\tau_{i}=\frac{M}{2 \pi h R_{i}^{2}}
$$

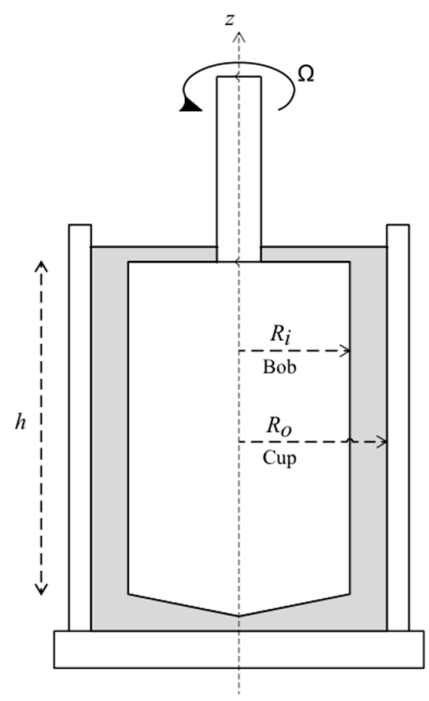

(a)

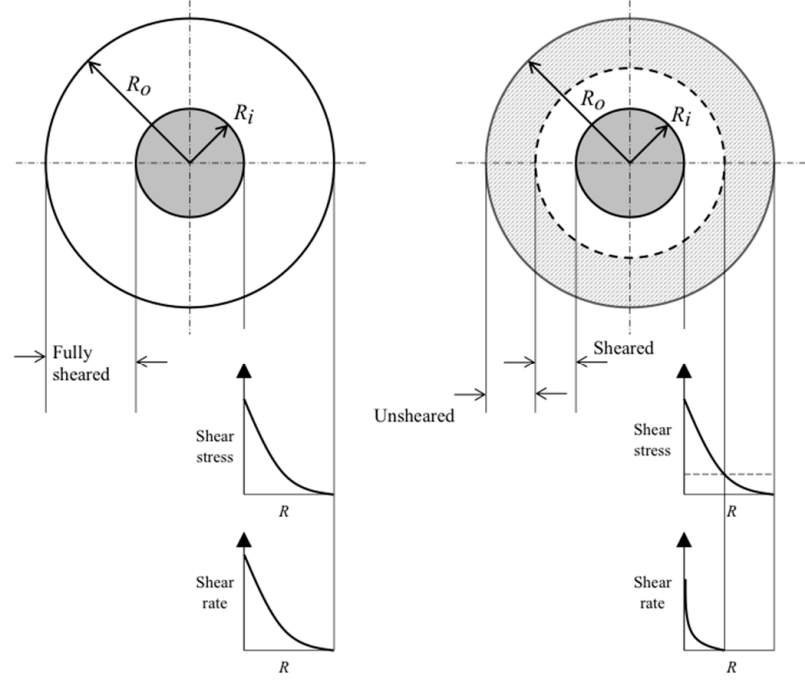

(b)

Figure 1. (a) Schematic representation of a Couette-flow Searle type rheometer. (b) Representing the shear-rate and shear-stress distribution for the fully and partially sheared flow modes.

Assuming a non-slip boundary condition (angular velocity is zero at the cup and at the bob is $\Omega$ ) and the flow being laminar and incompressible with no normal stresses, the shear rate $\dot{\gamma}$, the shear-stress $\tau$ and angular velocity $\Omega$, at the bob surface can be related as:

$$
\Omega=\int_{R_{i}}^{R_{o}} \frac{\dot{\gamma}(r)}{r} d r=\frac{1}{2} \int_{\tau_{o}}^{\tau_{i}} \frac{\dot{\gamma}(\tau)}{\tau} d \tau
$$

Solving Equation (3) yields in the conversion of angular velocity to the shear-rate thereby facilitating the derivation of the rheogram. The solution is dependent on the fluid behaviour $\dot{\gamma}(\tau)$, which translates to the rheological model of the fluid. Therefore, Equation (3) can be solved if the relation between shear-stress and shear-rate is known or an a priori assumption of the relation is required. In cases where this relation is unknown or cannot be assumed, the solution to Equation (3) becomes an ill-posed inverse problem. Another aspect complicating the solution to this integral, is the existence of partially sheared regions in the annulus, as seen in Figure 1b. For plastic materials, depending on the relative magnitude of the shear stress and the yield stress, $\tau_{y}$, two flow modes are possible: Partially Sheared Flow (PSF) and Fully Sheared Flow (FSF). Under the condition where $\tau_{i}<\tau_{y}<\tau_{0}$, only part of the fluid close to the bob is sheared while the remaining region is stationary (PSF) as illustrated in Figure 1b. When $\tau_{y}<\tau_{o}<\tau_{i}$, the shear stress everywhere in the flow exceeds 
the yield value and the fluid becomes sheared in the entire annulus (FSF mode). Considering all of these complications, there have been many procedures proposed to approximate the shear-rate. A review of these procedures have been provided in Estellé [23] and Chatzimina [20]. In this paper, the method used is one proposed by Yeow et al. [24]. This method uses Tikhonov regularisation to evaluate the shear-rates. This method was selected owing to the fact that it can cope with noised data, does not require a priori knowledge about the flow behaviour, can be used for fluids with a yield stress and can be applied to both fully and partially sheared modes.

\subsection{Tikhonov Regularisation}

Yeow and co-workers [22,24] have developed a procedure for solving Equation (3) by treating it as a Volterra integral equation of the first kind. The integral is solved using Tikhonov regularisation, which does not require the specification of the fluid behavior, and suppresses the propagation of eigenvalues related to 'noise' in the data when back mapping. The method followed a discretisation method and sought to derive the shear-rate by minimizing the fractional deviation between the computed shear-rate and its experimentally measured counterpart, the angular velocity. To ensure that the noise in the experimental data is not amplified, and to obtain smooth curves, a regularisation condition is imposed, which is minimising $\frac{\partial^{2} \dot{\gamma}(\tau)}{\partial \tau^{2}}$.

\subsubsection{Numerical Method}

The shear rate $\dot{\gamma}(\tau)$ that is to be estimated is discretised as $\dot{\gamma}=\left(\dot{\gamma}_{1}, \dot{\gamma}_{2}, \dot{\gamma}_{3} \ldots \dot{\gamma}_{N_{k}}\right)$ at uniformly spaced $\tau=\left(\tau_{1}=\tau_{\min }, \tau_{2}, \tau_{3} \ldots \tau_{N_{k}}=\tau_{\max }\right)$ and the equation that satisfies the condition to minimise the fractional deviation between the estimated shear-rate and the one experimentally measured and the regularisation condition is given by [24], shown in Equation (4).

$$
\dot{\gamma}=\left(A^{T} A+\lambda \beta^{T} \beta\right)^{-1} A^{T} I
$$

where $I$ is an appropriate identity matrix and $A$ is a coefficient matrix given by:

$$
A_{i j}=\frac{\frac{\alpha_{i j} \Delta \tau}{2 \tau_{j}}}{\Omega_{i}}, \quad \begin{aligned}
& i=1,2,3 \ldots N_{d} \\
& j=1,2,3 \ldots N_{k}
\end{aligned}
$$

where the number of discretisation points corresponds to $N_{k}$ and the number of measured points is $N_{d}$. $N_{k}$ is much larger than $N_{d}$ in implementation. The discretisation step $\Delta \tau$ in Equation (5) is given by:

$$
\Delta \tau=\frac{\tau_{\max }-\tau_{\min }}{N_{k}-1}
$$

and, $\alpha_{i j}$ is dependent on the numerical scheme used to approximate the integral in Equation (3). $\alpha_{i j}$ comprises the coefficient matrix from the numerical scheme, thereby using Simpson's $1 / 3$ rule to approximate the integral $\alpha_{i 1}=1 / 3, \alpha_{i j}=2 / 3$ for odd $j$ and $\alpha_{i 1}=4 / 3$ for even $j$.

The regularisation condition, minimising $\frac{\partial^{2} \dot{\gamma}(\tau)}{\partial \tau^{2}}$ gives rise to the tridiagonal matrix $\beta$ with $N_{k}$ rows in Equation (4).

$$
\beta=\left[\begin{array}{ccccccccc}
1 & -2 & 1 & & & & & & \\
& 1 & -2 & 1 & & & & & \\
\cdot & \cdot & . & . & . & . & . & . & . \\
\cdot & . & . & . & . & . & . & . & \cdot \\
\cdot & . & . & . & \cdot & . & . & . & \cdot \\
& & & & & 1 & -2 & 1 & \\
& & & & & & 1 & -2 & 1
\end{array}\right] \frac{1}{(\Delta \tau)^{2}}
$$


A regularisation parameter $\lambda$, shown in Equation (4), is used for the smoothing, which controls the extent to which the noise in the experiment is filtered at the cost of goodness of fit. As $\lambda$ depends on the noise in the data, number of data and discretisation points, and the numerical scheme; an iterative procedure is adopted to determine the appropriate $\lambda$ comparing the rheograms of a known non-Newtonian fluid. Further information on the derivation and implementation of the procedure can be found in the articles from Yeow [22,24].

\subsubsection{Recovering Yield Stress}

A slurry with yield stress $\tau_{y}$ will not flow or deform until the shear-stress applied on it is greater than its yield stress. Applying this knowledge to the integral in Equation (3), the lower limit becomes $\tau_{y}$. Also, the discretisation step is modified to:

$$
\Delta \tau=\frac{\tau_{\max }-\tau_{y}}{N_{k}-1}
$$

The yield stress of the material can be determined if at least one of the measured data points is in the partially sheared flow mode. This is done by using the condition:

$$
\dot{\gamma}\left(\tau_{y}\right)=0
$$

The $\tau_{y}$ is estimated iteratively using a Newton-Raphson like method, such that the $\dot{\gamma}(\tau)$ given by Equation (4) satisfies the condition mentioned in Equation (9).

\section{Methods and Materials}

\subsection{Experimental Procedure}

The experimental procedure was two fold: One which consists of validating the translation of angular velocity to shear rate from a wide gap rheometer and the other of obtaining the rheology of concentrated domestic slurry from a wide gap rheometer.

In validating the translation of angular velocity to shear rate from a wide gap rheometer, as seen in Section 2.2, a test fluid that could be operated both in a narrow gap and a wide gap rheometer was needed. In our case tomato ketchup was used. The rheology of the tomato ketchup was first obtained using a narrow gap rheometer. Using this rheology, the algorithm for translating the angular velocity was adjusted and validated by tuning the regularisation parameter $\lambda$. This tuning of the parameter was so done to match the rheology obtained from the wide gap to that from the narrow gap.

For the CDS rheology, a sample of concentrated domestic slurry was collected, as shown in Section 3.2. This sample was then prepared to various concentrations by gravity settling, and using the supernatant as a dilutent, shown in Section 3.3. Using the wide gap rheometer, as seen in Section 3.4, and the translation algorithm shown in Section 2.2, the rheograms were obtained. A Herschel-Bulkley model was used to represent these rheograms for practical purposes, and therefore the parameters for this model were obtained through parameter estimation procedures, as shown in Section 3.5.

\subsection{Materials}

The concentrated domestic slurry under study were collected from an experimental facility in the building of DeSaH B.V. in Sneek, the Netherlands. The facility has urine separation toilets fitted with a vacuum collection system. The samples collected consisted mostly of human faecal waste, toilet paper, and flushed water. The vacuum pump that was used to transport the waste slurry is fitted with a cutter. The samples were drawn from a collection tank downstream of the pump. To preserve the samples and retard any biological activity, they were transported to the lab immediately after collection in a cool box at $4{ }^{\circ} \mathrm{C} \pm 1{ }^{\circ} \mathrm{C}$. This procedure follows the advice given in [25]. The samples were kept in 
sealed containers to preserve the original moisture and avoid reactions with air. Once in the laboratory, the samples were tested within 1 day of their collection.

\subsection{Sample Preparation}

The samples collected were passed through a $2 \mathrm{~mm}$ mesh to remove coarse particles. The amounts of these coarse particles totalled 1 or 2 particles per $500 \mathrm{~mL}$ of slurry, which is considered negligible. This may be due to the presence of the cutter in the pump. Upon collection, the total suspended solids (TSS) in the sample was 2.6\% (wt./wt.). This was then concentrated to $8.4 \%$ (TSS wt./wt.), using gravity settling. To obtain samples of different concentrations the supernatant was used to dilute the concentrate.

The particle size distribution (PSD) of the sample is obtained to assess the requirement of the gap size for the rheometer. The PSD, presented in Figure 2, shows that the particles present in the sample vary in size from $1 \mu \mathrm{m}$ to about $2000 \mu \mathrm{m}$. The average D90 (representative particle size) of the sample is obtained using the cumulative size distribution, shown in Figure $2 \mathrm{~b}$, and is found to be $1108 \mu \mathrm{m}$. Therefore, to satisfy the continuum condition specified in Wazer [10], the gap size in the rheometer must be a minimum of $11 \mathrm{~mm}$.

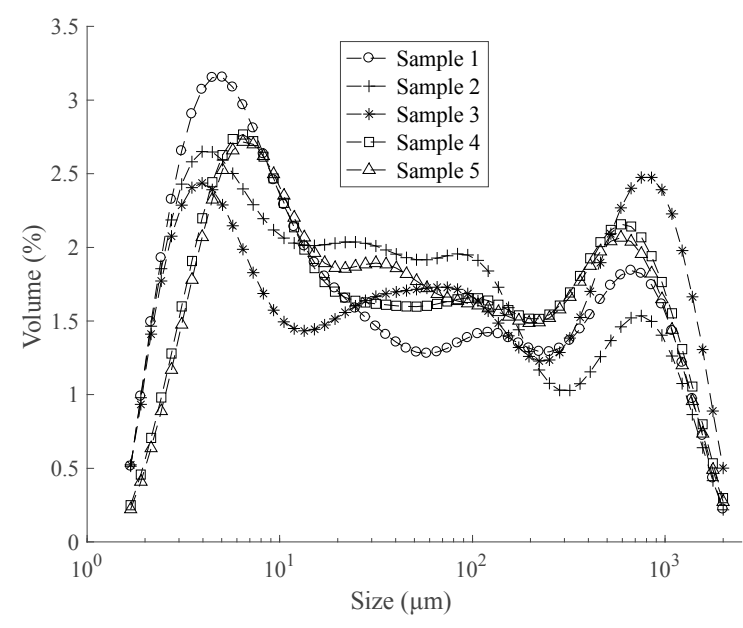

(a)

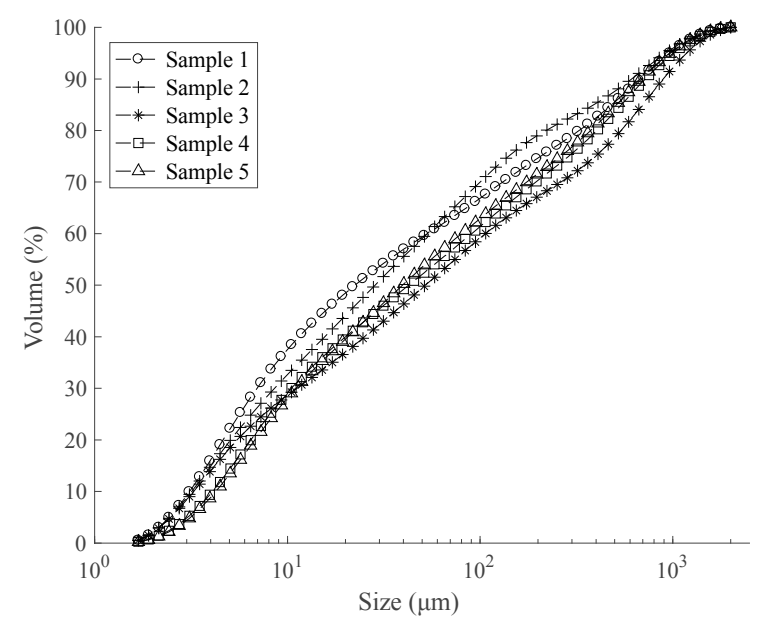

(b)

Figure 2. (a) Particle size distribution of 5 different samples from the collected concentrated domestic slurries (CDS) (b) Cumulative particle size distribution of the 5 samples.

\subsection{Wide Gap Rheometer}

The rheology measurements were performed with a MCR302 instrument from Anton Paar (Graz, Austria). Among the available bob and cup geometries, a bob (grooved) of diameter $45 \mathrm{~mm}$ and length $67.5 \mathrm{~mm}$ with a cup of diameter $74 \mathrm{~mm}$ is chosen. This gives a gap of the annulus to be $14.5 \mathrm{~mm}$, which is more the minimum required (specified in Section 3.3). During the measurements, a lid was used to cover the sample to avoid evaporation. For assuring the no-slip boundary condition mentioned in Section 2.1, the bob used is grooved laterally and a thin steel cage is attached to the cup. To ensure that all samples had the same initial condition, the sample is pre-sheared for $2 \mathrm{~min}$ at 500 RPM prior to each investigation and then let to rest for $1 \mathrm{~min}$. This procedure confirmed the repeatability of the tests. The torque was measured at various RPM (Rotation per minute) using a RPM ramp procedure. The wide RPM range was chosen so that the fluid is partially and fully sheared. The steady-state torque on the bob is recorded for every set RPM on the bob. With respect to settling during the measurements, at the macro level there was no supernatant observed which indicates no settling. To obtain the measurements for a particular concentration, 5 replicate tests were performed with different samples. 


\subsection{Model Parameter Estimation}

In this study, a genetic algorithm is used to estimate the parameters of the model representing their respective data [26]. This algorithm was chosen as it is a general algorithm, that is not tuned to suit a specific model. The advantage of this algorithm compared to the other usual gradient-based algorithms is that it has a low probability of being stuck in a local minimum and explores the parameter space well in general and is also able to deal with discontinuities in the goal function. More information about the implementation of the algorithm can be found in $[27,28]$. To evaluate the goodness of fit of the model to the data, a root mean square of the normalised residual (RMSNR, Equation (10)) value is used. Denoting that an RMSNR value close to 0 gives a very good fit.

$$
\operatorname{RMSNR}=\sqrt{\frac{\sum_{i=1}^{n}\left(\frac{\left(\left(y_{i}-\hat{y}_{i}\right)\right.}{y_{i}}\right)^{2}}{n}}
$$

where $y_{i}$ is the data for fitting, $\hat{y}_{i}$ is from the model and $n$ is the size of the data set. RMSNR gives the average proportionate deviation of the model from the data.

\section{Results and Discussion}

\subsection{Choosing $\lambda$}

Tomato ketchup, which has been commonly used by researchers as a test fluid, is also used here to demonstrate the validity of the method. For this purpose, the rheology of tomato ketchup from a narrow gap (the specifications of this set-up is detailed in [26]) is used to compare the results obtained from the wide gap geometry using Tikhonov regularisation shown in Figure 3. It is to be noted that the shear-rate vs. shear-stress curves obtained through a narrow gap approximation using a narrow gap $(R o / R i=1.08)$ rheometer is considered to be an accurate enough representation of the rheology of Tomato ketchup. As mentioned in Section 2.2, choosing a proper regularisation parameter is the bottle neck in the here used method. In this study, the regularisation parameter is chosen such that the deviation of the rheogram obtained by the method is less than $5 \%$ of that obtained from the narrow gap rheometer; this is shown in Figure 3. Thus, the obtained regularisation parameter is the one that is used throughout this paper for obtaining the rheograms of CDS. A systematic bias can be observed from the residuals between the rheograms obtained from that of the narrow gap and wide gap, as seen in Figure 3. For a perfect model these residues should show no autocorrelation or bias, but since the narrow gap results are approximated these biases arise.

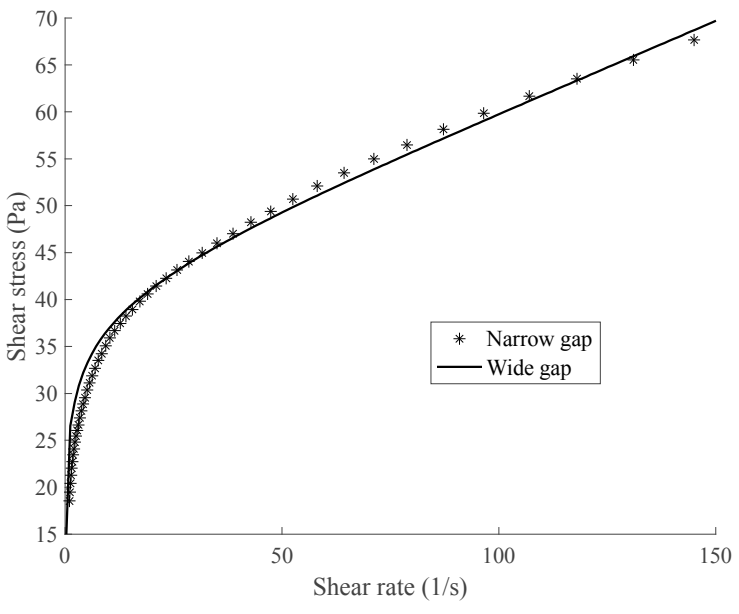

(a)

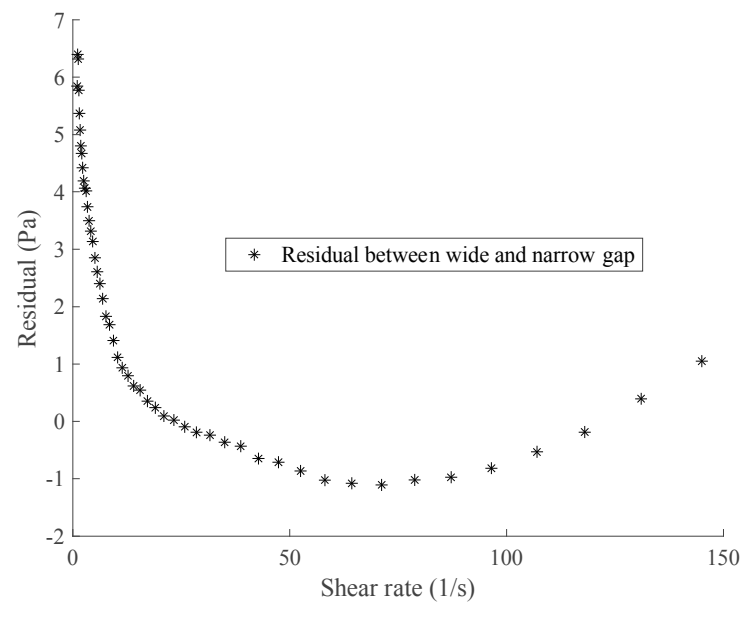

(b)

Figure 3. (a) Rheograms of Tomato ketchup obtained using both the narrow and wide gap geometry rheometers. (b) Residuals between the rheograms from wide and narrow gap. 


\subsection{CDS Rheograms}

Measurement data of shear-stress vs. angular velocity of the un-sieved slurry at various TSS concentrations are obtained using the wide gap rheometer described in Section 3.4. As can be seen from the measurement data in Figure 4, there is much noise present. This presence of the noise in the data has been a determining factor in choosing the Tikhonov regularisation method to obtain the rheograms. It can also be observed from Figure 4 that the initial points have a higher shear-stress as these points represent the partial shearing mode at lower angular velocities.

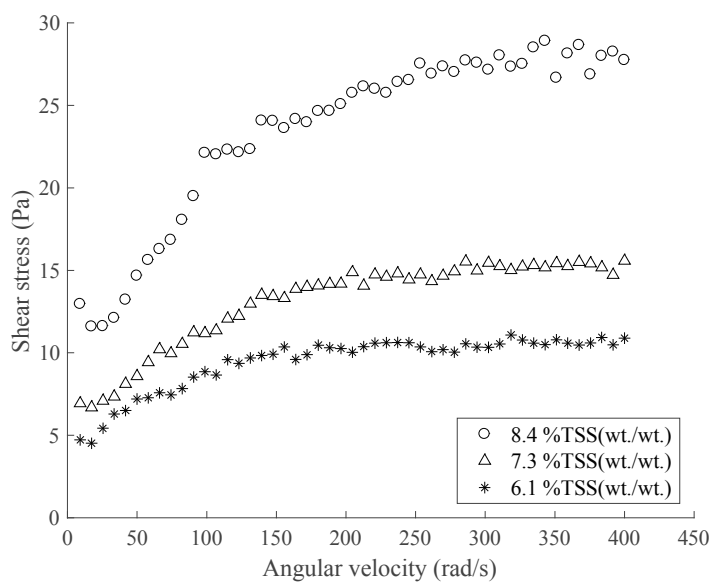

Figure 4. Shear-stress vs. angular velocity measurements for CDS using the wide gap geometry.

The rheograms (Figure 5) for the un-sieved CDS are obtained through the Tikhonov regularisation method, using the regularisation parameter determined in Section 4.1. From the iteration procedure mentioned in Section 2.2.2, the yield stress of the fluid is obtained. Having measured on a wide RPM range which also includes partially sheared flow facilitated the determination of the yield stress (Section 2.2.2). A Herschel-Bulkley rheological model, shown in Equation (11), is known to sufficiently represent the rheology of CDS [26]. This model will also be used in this paper. As the yield stress is already known, the remaining parameters $K$ and $n$ of the model are estimated using a genetic algorithm, shown in Section 3.5, with an RMSNR value of 0.02 which denotes a $2 \%$ deviation of the model from the data.

$$
\tau=\tau_{y}+K \dot{\gamma}^{n}
$$

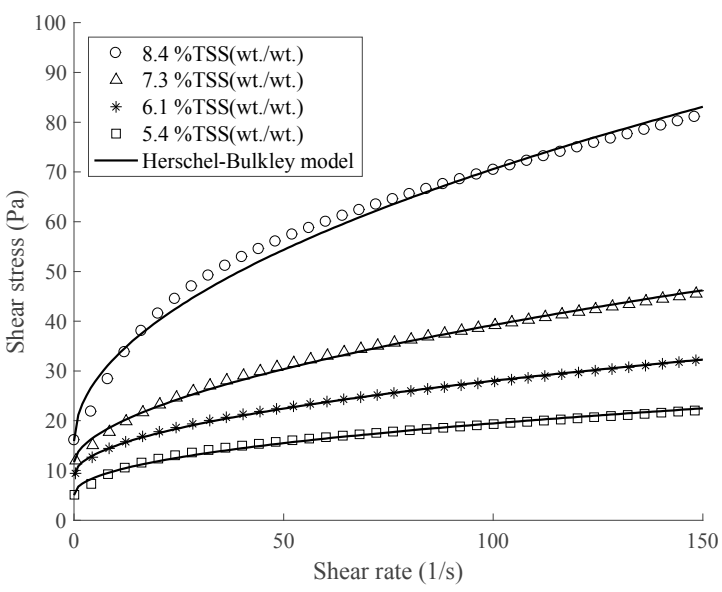

(a)

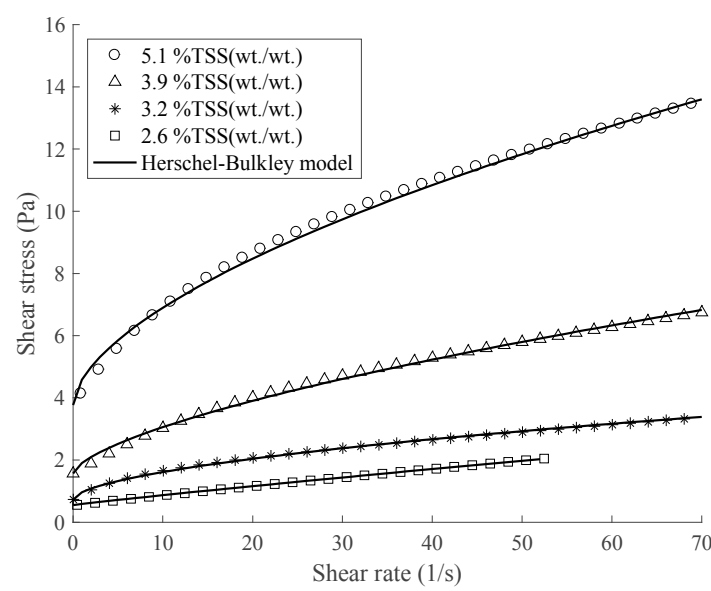

(b)

Figure 5. Rheograms of concentrated domestic slurries (CDS) obtained through Tikhonov regularization (a) Rheograms of concentration 5.4, 6.1, 7.3 and 8.4\% TSS (wt./wt.) (b) Rheograms of concentration 2.6, 3.9, 3.2 and 5.1\% TSS (wt./wt.). 


\subsection{Effect of TSS Concentration: C}

The influence of change in TSS concentration $C$ on the rheology of the slurry is expressed through the change in rheological parameters. These are found to be similar to the ones already published $[9,15,29]$.

\subsubsection{Yield Stress: $\tau_{y}$}

The minimum shear stress that is required to overcome the flow resistance for the slurry to start flowing is the yield stress. The yield stress of the slurry when plotted against the concentration of TSS, as seen in Figure 6, shows an exponentially increasing behaviour. Although the same has been reported by some researchers $[9,29]$, in general the models used represent the yield stress vary largely. This is predominantly due to the fact that the model is built to only empirically represent the data abandoning physical reasoning. The same is true for the other parameters $K$ and $n$ as well. That being said, there is not much information about these parameters to give them a physical dimension, as they are in themselves an empirical representation of the rheological data. Here an attempt is made to give a semi-empirical representation to the influence of TSS concentration to the rheological parameters $\tau_{y}$, $K$ and $n$. This is so done by setting boundaries for these parameters, for which an explanation follows.

The increasing behaviour of the yield stress with the increase in concentration can be characterised through the particle interactions. Albeit these interactions are weak physical forces, the increase in concentration increases the number of neighbouring particles. This causes the formation of particle structure, where the yield stress is applied to break and overcome it. As mentioned in previous works [26], the yield stress is 0 at low concentrations and only above a certain concentration does it aquire a physical plausible value. This threshold concentration is known approximately to be around 3.0\% TSS (wt./wt.) [26]. The model so chosen to represent the yield stress accounts for all this information and is given in Equation (12) (with a RMSNR value of 0.25). This is chosen so that the value of yield stress reduces to 0 at concentration $0 \%$ TSS, as the slurry reduces to just water and above $2.0 \%$ TSS (wt./wt.) (a parameter found through model fitting) the presence of yield stress is established.

$$
\begin{array}{cl}
\tau_{y}=0.71(C-2.0)^{1.7} & \text { for } C>2.0 \% \text { TSS }(w t . / w t .) \\
\tau_{y}=0 & \text { for } C<2.0 \% \text { TSS }(w t . / w t .)
\end{array}
$$

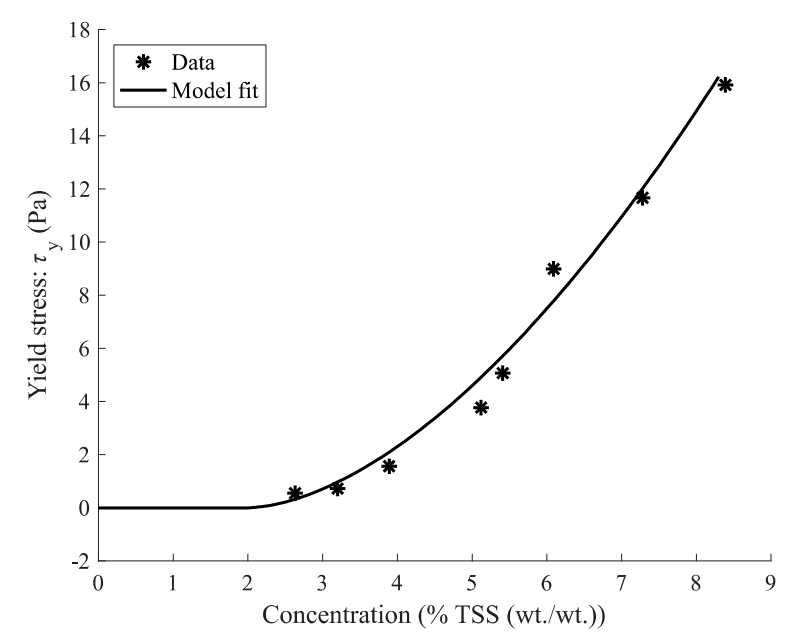

Figure 6. Influence of change in concentration of \% total suspended solids (TSS) to Yield stress $\tau_{y}$ of concentrated domestic slurries (CDS).

\subsubsection{Consistency Index: $K$}

The consistency index $K$ that represents the inherent flow viscosity in the slurry also increases exponentially, as seen in Figure 7, with increasing TSS concentration $C$ and is represented using the Equation (13). The latter differs with Equation (12) used to represent the yield stress as the boundary 
condition for Equation (13) is different. As the concentration approaches 0, the consistency index reduces to a low value close to that of water, but will not become 0 . This information is used to derive Equation (13) representing $K$ with a RMSNR of 0.32 . This has been suggested by other researchers as well $[9,29]$.

$$
K=0.07 e^{0.5 C}
$$

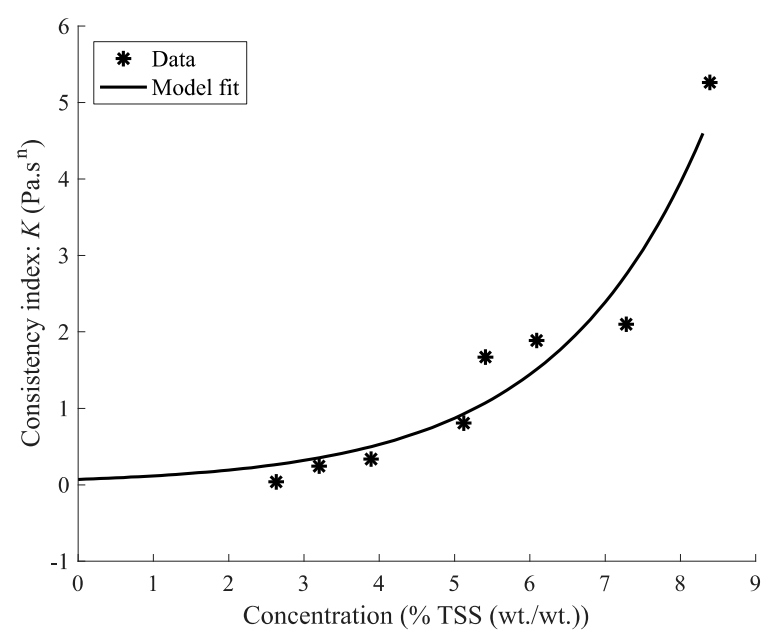

Figure 7. Influence of change in concentration of \% total suspended solids (TSS) to consistency index $K$ of concentrated domestic slurries (CDS).

\subsubsection{Behaviour Index: $n$}

The behaviour index $n$, represents the extent of shear thinning nature of the slurry. From Figure 8 , that shows the influence of TSS concentration on behaviour index, it is seen that $n$ decreases from 1 at $0 \%$ TSS to a lower value at higher concentrations and that the change in the behaviour index is observed only after a threshold concentration (also observed in the many models reviewed by Seyssiecq et al. [29]). Above this threshold, the behaviour index is observed to decrease with an inverse power law. To model this nature, Equation (14) (RMSNR 0.11) is used to represent the behaviour index and this model has been used by various other researchers $[9,29]$. Using this model, sshown in Equation (14), the threshold concentration is found to be 2.6\% TSS (wt./wt.), estimated as a parameter in the model. As discussed earlier, most flow characteristics of these slurries can be attributed to the fluid particle structures. The concept of structural units (SUs) vis-à-vis fluid particle structures introduced by Quemada [30] expresses the view that the rheological model is essentially based on the concept of effective volumes of these SUs . As for the shear thinning behaviour, it is particularly associated with the alignment of fluid particles in the direction of flow, after breaking to its constituent particles from being a composite fluid structure. As the shear-rate increases, the amount of structures broken increases, and with the particles aligning with the flow cause the shear thinning behaviour i.e., easiness to flow behaviour. Also, as can be seen in Figure 8, the behaviour index tends to reach a plateau. Thereby, it can be hypothesised that the decrease in the flow behaviour index with increase in concentration although being significant in the beginning, will reach a plateau at very high concentration. The plateau at high concentration can be associated with the low significance of the fluid structures broken to that of particle-particle interaction. That is to say, although there might be a significant portion of the fluid structures broken and aligned along the flow, the inherent particle-particle interaction certainly outplays at higher concentration thereby lowering the effect of broken fluid structures.

$$
\begin{array}{cc}
n=0.65-0.09 \log (C-2.57) & \text { for } C>2.6 \% \text { TSS (wt. } / \text { wt. }) \\
n=1 & \text { for } C<2.6 \% \text { TSS (wt. } / \text { wt. })
\end{array}
$$




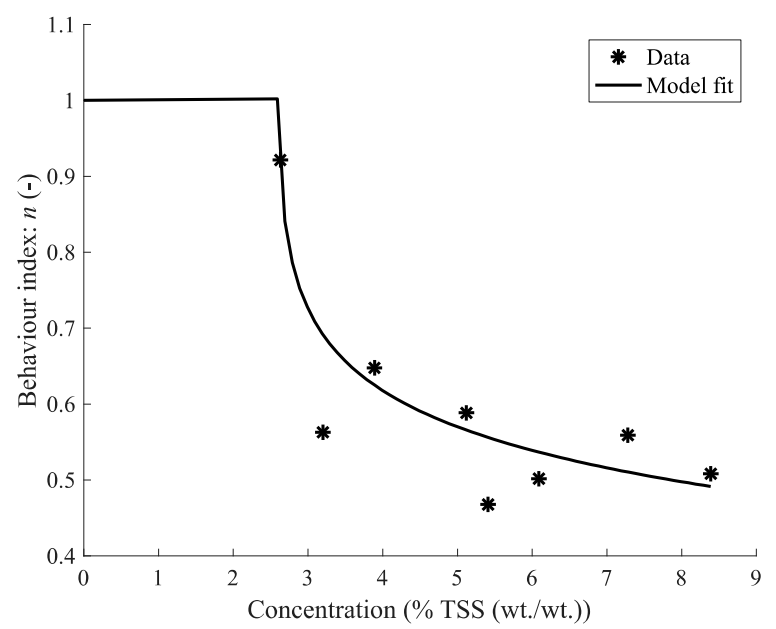

Figure 8. Influence of change in concentration of \% total suspended solids (TSS) to behaviour index $n$ of concentrated domestic slurries (CDS).

\subsection{Comparison with Other Wastewater Slurries}

Slurries encountered in the different stages of wastewater treatment processes have been known to behave as non-Newtonian slurries [29]. On comparing these slurries with CDS (Figure 9) it can be seen that they have similar trends of behaving as a shear thinning slurry, and with similar tendencies to the change in concentration. In particular, on comparing the model equations of the consistency index $K$ and yield stress $\tau_{y}$ with the ones proposed of Markis et al. [31], it is seen that the model equations and the values of the parameters are quite similar (although the values for the yield stress are higher). This again elucidates that the slurries in comparison behave similarly.

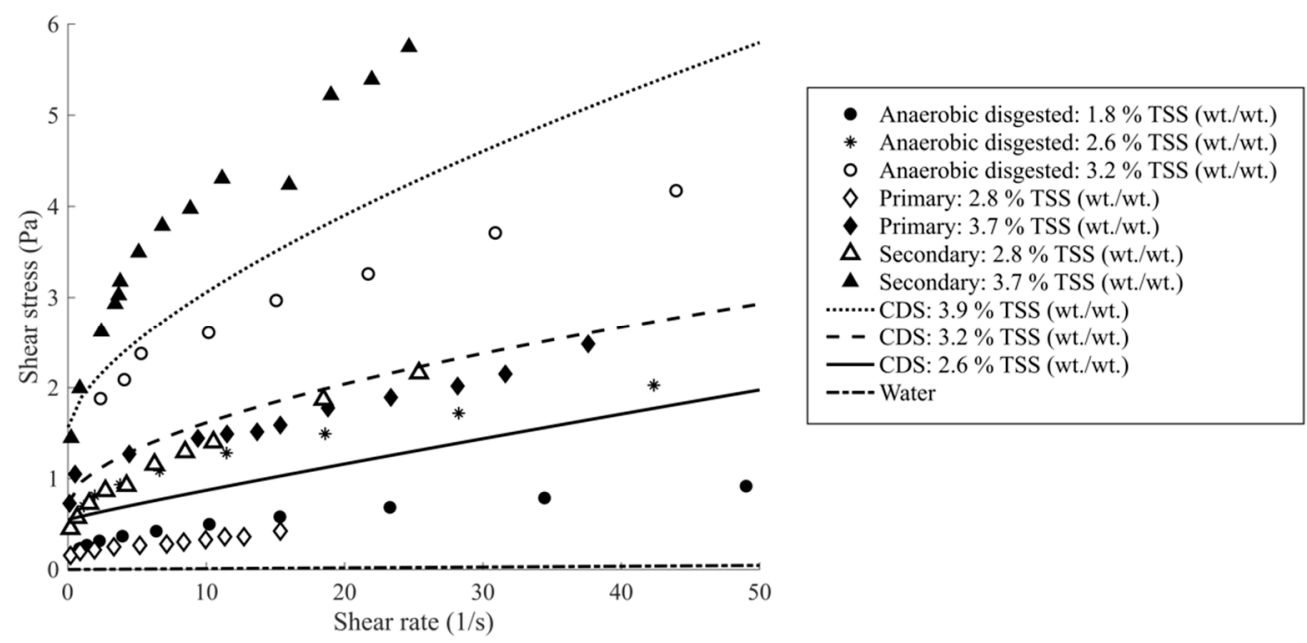

Figure 9. Comparison of the rheograms of concentrated domestic slurries (CDS) (this study) and similar wastewater sludges: Anaerobic digested [32], primary and secondary sludge [31].

\section{Conclusions}

The rheology of CDS is fundamental in designing transport systems to carry them. A rotating rheometer is commonly used for this purpose. The geometry of the rotating rheometer to be used is highly dependent on the size of the particles in the slurry. As the slurry has large particles present in them, the choice of geometry is crucial. For this purpose, the rheology of un-sieved concentrated domestic slurry is measured using a wide gap rheometer. To obtain the shear-rates from a wide gap rotating rheometer it is essential to choose an appropriate mathematical method, and for this purpose 
a Tikhonov regularisation method is used. This particular method (Tikhonov regularisation) is chosen as it copes with the noisy data and yield stress present in the fluid. This method is validated using a test fluid, tomato ketchup, and the regularisation parameter (essential to the method) is obtained using the same fluid. The obtained rheology of the un-sieved slurry presents a complete information on the flow characteristics of concentrated domestic slurry. This complete information is crucial in providing design specification of slurry handling systems. A Herschel-Bulkley rheological model is used to represent the rheology of the slurry. The influence of change in concentration of TSS on the rheology is studied through its influence on the parameters of the rheological model. The yield stress and consistency index of the Herschel-Bulkley model increase exponentially and are represented through their respective exponential models. Similarly, an attempt has been made to represent the behaviour index through an inverse power law model. It is observed that the yield stress in the slurry is only physically meaningful above $2 \%$ TSS (wt./wt.) and the behaviour index above $2.6 \%$ TSS (wt./wt.). These equations provide practitioners with usable forms of information to design slurry handling systems.

Author Contributions: A.K.T.R. conceived the experimental design, performed the experiments, sorted the theoretical background, implemented the mathematical models and wrote the manuscript. F.C. and J.v.L. provided guidance and supervision during all the process.

Funding: This project is funded by NWO-domain TTW, grant number 13347.

Acknowledgments: This R\&D project is carried out by Delft University of Technology and funded by NWO-domain TTW, Deltares, Stowa, RIONED, Waternet, Waterboard Zuiderzeeland, Sweco and XYLEM. We acknowledge the support of the funding bodies and their contributions to thie research.

Conflicts of Interest: The authors declare no conflicts of interest.

\section{References}

1. Slatter, P.T. Transitional and Turbulent Flow of Non-Newtonian Slurries in Pipes. Ph.D. Thesis, University of Cape Town, Cape Town, South Africa, 1995.

2. Thomas, A.D.; Wilson, K.C. New analysis of non-newtonian turbulent flowndashyield-power-law fluids. Can. J. Chem. Eng. 1987, 65, 335-338. [CrossRef]

3. Chilton, R.; Stainsby, R.; Thompson, S. The design of sewage sludge pumping systems. J. Hydraul. Res. 1996, 34, 395-408. [CrossRef]

4. Chhabra, R.P.; Richardson, J.F. Non-Newtonian Flow and Applied Rheology: Engineering Applications; Butterworth-Heinemann: Oxford, UK, 2011; ISBN 0080951600.

5. AL-Behadili, A.J.M.; Sellier, M.; Nokes, R.; Moyers-Gonzalez, M.; Geoghegan, P.H. Rheometry based on free surface velocity. Inverse Probl. Sci. Eng. 2018, 1-21. [CrossRef]

6. Longo, S.; Di Federico, V.; Archetti, R.; Chiapponi, L.; Ciriello, V.; Ungarish, M. On the axisymmetric spreading of non-Newtonian power-law gravity currents of time-dependent volume: An experimental and theoretical investigation focused on the inference of rheological parameters. J. Nonnewton. Fluid Mech. 2013, 201, 69-79. [CrossRef]

7. Di Federico, V.; Longo, S.; King, S.E.; Chiapponi, L.; Petrolo, D.; Ciriello, V. Gravity-driven flow of Herschel-Bulkley fluid in a fracture and in a 2D porous medium. J. Fluid Mech. 2017, 821, 59-84. [CrossRef]

8. Gurung, A.; Haverkort, J.W.; Drost, S.; Norder, B.; Westerweel, J.; Poelma, C. Ultrasound image velocimetry for rheological measurements. Meas. Sci. Technol. 2016, 27, 94008. [CrossRef]

9. Eshtiaghi, N.; Markis, F.; Yap, S.D.; Baudez, J.-C.; Slatter, P. Rheological characterisation of municipal sludge: A review. Water Res. 2013, 47, 5493-5510. [CrossRef] [PubMed]

10. Van Wazer, J.R. Viscosity and Flow Measurement: A Laboratory Handbook of Rheology; Interscience Publishers: Geneva, Switzerland, 1963.

11. Slatter, P.T. The rheological characterisation of sludges. Water Sci. Technol. 1997, 36, 9-18. [CrossRef]

12. Dick, R.I.; Ewing, B.B. The rheology of activated sludge. J. Water Pollut. Control Fed. 1967, 543-560.

13. Koseoglu, H.; Yigit, N.O.; Civelekoglu, G.; Harman, B.I.; Kitis, M. Effects of chemical additives on filtration and rheological characteristics of MBR sludge. Bioresour. Technol. 2012, 117, 48-54. [CrossRef] [PubMed] 
14. Mori, M.; Seyssiecq, I.; Roche, N. Rheological measurements of sewage sludge for various solids concentrations and geometry. Process Biochem. 2006, 41, 1656-1662. [CrossRef]

15. Ratkovich, N.; Horn, W.; Helmus, F.P.; Rosenberger, S.; Naessens, W.; Nopens, I.; Bentzen, T.R. Activated sludge rheology: A critical review on data collection and modelling. Water Res. 2013, 47, 463-482. [CrossRef] [PubMed]

16. Battistoni, P. Pre-treatment, measurement execution procedure and waste characteristics in the rheology of sewage sludges and the digested organic fraction of municipal solid wastes. Water Sci. Technol. 1997, 36, 33-41. [CrossRef]

17. Battistoni, P.; Pavan, P.; Mata-Alvarez, J.; Prisciandaro, M.; Cecchi, F. Rheology of sludge from double phase anaerobic digestion of organic fraction of municipal solid waste. Water Sci. Technol. 2000, 41, 51-59. [CrossRef] [PubMed]

18. Woolley, S.M.; Cottingham, R.S.; Pocock, J.; Buckley, C.A. Shear rheological properties of fresh human faeces with different moisture content. Water SA 2014, 40, 273-276. [CrossRef]

19. Ancey, C. Solving the Couette inverse problem using a wavelet-vaguelette decomposition. J. Rheol. (N. Y.) 2005, 49, 441-460. [CrossRef]

20. Chatzimina, M.; Gerogiou, G.; Alexandrou, A. Wall Shear Rates in Circular Couette Flow of a Herschel-BulkleyFluid. Appl. Rheol. 2009, 19, 34288.

21. Krieger, I.M.; Elrod, H. Direct Determination of the Flow Curves of Non-Newtonian Fluids. II. Shearing Rate in the Concentric Cylinder Viscometer. J. Appl. Phys. 1953, 24, 134-136. [CrossRef]

22. Leong, Y.K.; Yeow, Y.L. Obtaining the shear stress shear rate relationship and yield stress of liquid foods from Couette viscometry data. Rheol. Acta 2003, 42, 365-371. [CrossRef]

23. Estellé, P.; Lanos, C.; Perrot, A. Processing the Couette viscometry data using a Bingham approximation in shear rate calculation. J. Nonnewton. Fluid Mech. 2008, 154, 31-38. [CrossRef]

24. Yeow, Y.L.; Ko, W.C.; Tang, P.P.P. Solving the inverse problem of Couette viscometry by Tikhonov regularization. J. Rheol. 2000, 44, 1335-1351. [CrossRef]

25. Association, A.P.H. Standard Methods for the Examination of Water and Wastewater; American Public Health Association (APHA): Washington, DC, USA, 2005.

26. Thota Radhakrishnan, A.; van Lier, J.; Clemens, F. Rheological characterisation of concentrated domestic slurry. Water Res. 2018, 141, 235-250. [CrossRef] [PubMed]

27. Chaudhuri, A.; Wereley, N.M.; Radhakrishnan, R.; Choi, S.B. Rheological parameter estimation for a ferrous nanoparticle-based magnetorheological fluid using genetic algorithms. J. Intell. Mater. Syst. Struct. 2006, 17, 261-269. [CrossRef]

28. Rooki, R.; Ardejani, F.D.; Moradzadeh, A.; Mirzaei, H.; Kelessidis, V.; Maglione, R.; Norouzi, M. Optimal determination of rheological parameters for herschel-bulkley drilling fluids using genetic algorithms (GAs). Korea-Aust. Rheol. J. 2012, 24, 163-170. [CrossRef]

29. Seyssiecq, I.; Ferrasse, J.-H.; Roche, N. State-of-the-art: Rheological characterisation of wastewater treatment sludge. Biochem. Eng. J. 2003, 16, 41-56. [CrossRef]

30. Quemada, D. Rheological modelling of complex fluids. I. The concept of effective volume fraction revisited. Eur. Phys. J. Appl. Phys. 1998, 1, 119-127. [CrossRef]

31. Markis, F.; Baudez, J.-C.; Parthasarathy, R.; Slatter, P.; Eshtiaghi, N. Rheological characterisation of primary and secondary sludge: Impact of solids concentration. Chem. Eng. J. 2014, 253, 526-537. [CrossRef]

32. Baudez, J.C.; Markis, F.; Eshtiaghi, N.; Slatter, P. The rheological behaviour of anaerobic digested sludge. Water Res. 2011, 45, 5675-5680. [CrossRef] [PubMed]

(C) 2018 by the authors. Licensee MDPI, Basel, Switzerland. This article is an open access article distributed under the terms and conditions of the Creative Commons Attribution (CC BY) license (http:// creativecommons.org/licenses/by/4.0/). 